\title{
APLICAÇÃO DO NURSING ACTIVITIES SCORE EM PACIENTES DE ALTA DEPENDÊNCIA DE ENFERMAGEM ${ }^{1}$ APPLICATION OF THE NURSING ACTIVITIES SCORE IN PATIENTS WITH HIGH DEPENDENCY ON NURSING CARE APLICACIÓN DEL NURSING ACTIVITIES SCORE EN PACIENTES DE ALTA DEPENDENCIA DE ENFERMERÍA
}

\author{
Marian Keiko Frossard Lima², Rosângela Tsukamoto³, Fernanda Maria Togeiro Fugulin ${ }^{4}$
}

\footnotetext{
${ }^{1}$ Trabalho extraído da monografia apresentada ao Curso de Especialização em Gerenciamento dos Serviços de Saúde da Escola de Enfermagem da Universidade de São Paulo (USP).

${ }^{2}$ Especialista em Gerenciamento de Serviços de Saúde. Enfermeira do Instituto do Coração do Hospital das Clínicas da Faculdade de Medicina da USP. São Paulo, Brasil.

${ }^{3}$ Especialista em Gerenciamento de Serviços de Saúde. Enfermeira do Hospital Universitário da USP. São Paulo, Brasil.

${ }^{4}$ Doutora em Enfermagem. Professora do Departamento de Orientação Profissional da Escola de Enfermagem da USP. Orientadora da monografia. São Paulo, Brasil.
}

PALAVRAS-CHAVE: Carga de trabalho. Administração de recursos humanos. Gerenciamento do tempo. Enfermagem. Cuidados de enfermagem.

KEYWORDS: Workload Personnel management. Time management. Nursing. Nursing care.
RESUMO: Este estudo, do tipo exploratório-descritivo, teve por objetivos avaliar a aplicabilidade do Nursing Activities Score como instrumento de medida da carga de trabalho na assistência aos pacientes da categoria de cuidados alta dependência de enfermagem e relacionar a pontuação média obtida com os tempos médios de assistência preconizados pelo Conselho Federal de Enfermagem. A coleta de dados para a determinação da pontuação do Nursing Activities Score ocorreu no período de 13 de julho a 01 de agosto de 2007, por meio de consulta aos prontuários dos pacientes internados na enfermaria de alta dependência de uma Unidade de Clínica Médica. A análise dos dados foi realizada mediante estatística descritiva e medida de variabilidade. O instrumento demonstrou ser aplicável a esses pacientes, sendo necessário o estabelecimento de diretrizes para a sua aplicação. Concluiu-se que o paciente classificado como alta dependência de enfermagem necessita, em média, de 12,3 horas de assistência nas 24 horas.

ABSTRACT: The objectives of this exploratory-descriptive study were to evaluate the applicability of the Nursing Activities Score as an instrument to measure workload in caring for patients classified into the category of high dependency on nursing care and to correlate the obtained mean scores with mean time of assistance proclaimed by the Brazilian Federal Nursing Council. Data was collected to determine the Nursing Activities Score in the period from July 13th to August 1, 2007 through means of consulting the patient charts of those interned in the high dependency ward of an out-patient clinic of a Medical Clinical Unit. Data analysis was performed by descriptive statistics and measure of variability. The instrument was effective for these patients, while the establishment of guidelines was needed for its application. It was concluded that the patient classified as high dependant on nursing care needs on average of 12.3 hours of assistance within a 24-hour period.
PALABRAS CLAVE: Carga de trabajo. Administración de personal. Administración del tiempo. Enfermería. Atención de enfermería.
RESUMEN: Estudio de tipo exploratorio-descriptivo, cuyos objetivos fueron: evaluar la aplicabilidad del Nursing Activities Score como instrumento de medida de la carga de trabajo en la asistencia a los pacientes de la categoría de cuidados de alta dependencia de enfermería, y además, relacionar la puntuación media obtenida con los tiempos medios de asistencia establecidos por el Consejo Federal de Enfermería. La recolección de los datos fue hecha en el período de 13 de julio a 1 de agosto de 2007, por medio de la consulta a los prontuarios de los pacientes internados en la enfermería de alta dependencia de una Unidad de Clínica Médica. El análisis de los datos se realizó por estadística descriptiva y por la medida de la variabilidad. El instrumento de análisis mostró ser eficaz para esos pacientes, siendo necesario el establecimiento de directrices para su aplicación. Este estudio concluyó que el paciente clasificado como de alta dependencia de enfermería necesita una media de 12,3 horas de asistencia, en un período de 24 horas.
Fernanda Maria Togeiro Fugulin

Endereço: Av. Dr. Enéas de Carvalho Aguiar, 419 05403-000 - Cerqueira César, São Paulo, SP, Brasil E-mail: ffugulim@usp.br
Artigo original: Pesquisa Recebido em: 15 de abril de 2008 Aprovação final: 17 de novembro de 2008 


\section{INTRODUÇÃO}

As organizações de saúde brasileiras, principalmente as instituições hospitalares públicas, têm se caracterizado por uma sobrecarga de trabalho dos profissionais de enfermagem, devido à insuficiência quantitativa e qualitativa de trabalhadores frente à demanda assistencial da clientela atendida, que interferem, diretamente, na eficácia e na qualidade dos serviços oferecidos.

Diante desse cenário, a temática dimensionamento de pessoal de enfermagem assume importante significado, na medida em que procura adequar o quadro de pessoal disponível às necessidades assistenciais da clientela, aos objetivos institucionais e às expectativas dos clientes internos e externos. ${ }^{1}$

Uma das principais etapas dos métodos de dimensionamento de pessoal de enfermagem refere-se à identificação da carga de trabalho da unidade de assistência de enfermagem, compreendida como o produto da quantidade média de pacientes assistidos, segundo o grau de dependência da equipe de enfermagem, pelo tempo médio de assistência utilizada, por paciente, de acordo com o grau de dependência. ${ }^{2}$

Dessa forma, verifica-se que, para a identificação dessa variável, é necessário conhecer o grau de dependência dos pacientes em relação à equipe de enfermagem, por meio da adoção de um Sistema de Classificação de Pacientes (SCP), bem como determinar o tempo médio de assistência utilizado para o atendimento de suas necessidades.

No Brasil, o Conselho Federal de Enfermagem (COFEN), ao estabelecer critérios para o dimensionamento do quadro de profissionais de enfermagem nas instituições de saúde, ${ }^{3}$ referendou o SCP desenvolvido na Unidade de Clínica Médica do Hospital Universitário da USP (HU-USP), que estabelece cinco categorias de cuidados, de acordo com a dependência do paciente em relação à assistência de enfermagem: cuidados intensivos, cuidados semi-intensivos, cuidados alta dependência, cuidados intermediários e cuidados mínimos. ${ }^{4}$ Entretanto, o COFEN não considerou a categoria de cuidados de alta dependência de enfermagem, que se refere aos pacientes crônicos, que apresentam total dependência de enfermagem para o atendimento de suas necessidades humanas básicas.

Com base neste $\mathrm{SCP},{ }^{4}$ a Resolução COFEN $N^{\circ}$ 293/04 indicou as horas mínimas de assistência e, também, a distribuição percentual dos profissionais de enfermagem, para cada tipo de cuidado. ${ }^{3}$
No que diz respeito às necessidades assistenciais do paciente crônico, a Resolução COFEN determinou que ao paciente crônico com mais de 60 anos, sem acompanhante, classificado nas categorias de cuidado intermediário ou semiintensivo, deverá ser acrescido 0,5 horas de assistência àquelas estabelecidas para as categorias de cuidado correspondentes. ${ }^{3}$

Entretanto, na prática, verifica-se que a falta de parâmetros próprios para o dimensionamento de pessoal de enfermagem, que contemple os pacientes classificados nesta categoria de cuidados, faz com que esses pacientes sejam classificados de forma equivocada provocando, conseqüentemente, distorções na previsão do quantitativo de pessoal de enfermagem. ${ }^{5}$

Frente a essa realidade torna-se necessário a proposição e a validação de parâmetros relacionados ao tempo de assistência de enfermagem utilizado para o atendimento desta clientela. Diante das dificuldades instrumentais e operacionais para a realização desse procedimento, considera-se que uma nova geração de instrumentos, desenvolvidos no cenário internacional com a proposta de quantificar a carga de trabalho de enfermagem e avaliar a adequação quantitativa de pessoal para o atendimento das necessidades assistenciais dos pacientes internados em Unidades de Terapia Intensiva (UTIs), podem ser testados e validados na realidade dos pacientes de alta dependência de enfermagem.

Dentre esses instrumentos, o Nursing Activities Score (NAS) tem sido indicado como um instrumento sensível e promissor para dimensionar a carga de trabalho da equipe de enfermagem em UTI, ${ }^{6-8}$ por possibilitar a identificação do tempo de assistência de enfermagem dispensando aos pacientes internados nestas unidades, subsidiando, desta forma, o cálculo e a distribuição dos profissionais de enfermagem.

A soma das pontuações obtidas com a avaliação retrospectiva do paciente, frente a cada item do instrumento, representa o quanto do tempo de trabalho de um profissional o paciente requereu nas últimas 24 horas. Assim, uma pontuação igual a 100 pontos, por exemplo, significa que o paciente requereu $100 \%$ do tempo de um trabalhador de enfermagem no seu cuidado, nas últimas 24 horas. ${ }^{7-8}$

A pontuação do NAS foi correlacionada com o tempo de assistência prestada, indicando que cada ponto NAS equivale a 14,4 minutos. ${ }^{9}$

Assim, visando contribuir para a superação das dificuldades relacionadas à inexistência de pa- 
râmetros específicos para a previsão do quadro de pessoal de enfermagem para assistir aos pacientes classificados como alta dependência de enfermagem, este estudo tem a finalidade de verificar a adequação da aplicação do NAS aos pacientes classificados nesta categoria de cuidados.

\section{OBJETIVOS}

Avaliar a aplicabilidade do NAS, como instrumento de medida da carga de trabalho de enfermagem na assistência, aos pacientes de alta dependência de enfermagem;

Relacionar a pontuação média obtida com os tempos médios de assistência preconizados pelo COFEN.

\section{METODOLOGIA}

\section{Local do Estudo}

O estudo, de abordagem quantitativa, do tipo exploratório-descritivo, foi desenvolvido na Enfermaria de Alta Dependência (EAD) da Unidade de Clínica Médica (UCM) do HU-USP, cujo serviço está organizado de acordo com o SCP, implantado desde 1990. .,10 $^{-10}$

O HU-USP tem por finalidade promover o ensino, a pesquisa e a extensão de serviços de saúde à comunidade. Presta assistência hospitalar de média complexidade e atende as quatro especialidades básicas: médica, cirúrgica, obstetrícia e pediatria.

A UCM do HU-USP dispõe de 44 leitos, assim distribuídos: 14 leitos para Cuidados Alta Dependência; 22 leitos para Cuidados Intermediários e oito leitos para Cuidados Mínimos.

O gerenciamento dos leitos é realizado pelas enfermeiras da unidade. Os pacientes são internados nos leitos correspondentes às suas necessidades de cuidado, observando-se o grau de dependência da equipe de enfermagem, de acordo com o Sistema de Classificação de Pacientes referendado pelo COFEN, já consolidado entre os profissionais da área. ${ }^{10}$ Diariamente, as enfermeiras avaliam e classificam todos os pacientes internados, remanejando-os, quando necessário, para o leito correspondente ao seu perfil assistencial.

Os 14 leitos destinados aos pacientes de alta dependência estão distribuídos em seis quartos individuais utilizados, preferencialmente, para pacientes que necessitam de isolamento ou que permanecem com acompanhante, e uma enfer- maria com oito leitos, sendo quatro femininos e quatro masculinos.

A EAD possibilita a visualização de todos os pacientes internados. Conta com um posto de enfermagem onde estão disponibilizados todos os materiais de consumo e medicamentos utilizados pelos pacientes, visando à otimização do atendimento. Nesses leitos são admitidos os pacientes classificados como cuidados alta dependência de enfermagem, que apresentam total dependência das ações de enfermagem para o atendimento das necessidades humanas básicas e que não contam com a presença de um acompanhante ou cuidador.

\section{Amostra}

Participaram do estudo todos os pacientes classificados como alta dependência de enfermagem, internados na EAD da UCM do HU-USP, no período de 13/07/2007 a 01/08/2007, independente do número de dias de internação, constituindo uma amostra não probabilística, intencional.

\section{Procedimentos de Coleta de Dados}

Após a aprovação pela Comissão de Ensino e Pesquisa e pelo Comitê de Ética em Pesquisa do HU-USP, sob o Registro No 742/ 07 - SISNEP CAAE: 0030.0.198.198-07, a coleta de dados para a determinação do NAS foi realizada de forma sistematizada, por uma das pesquisadoras, enfermeira da UCM, uma vez ao dia, durante 20 dias consecutivos, por meio de consulta retrospectiva aos prontuários dos pacientes internados, sendo liberada a assinatura do Termo de Consentimento Livre e Esclarecido.

Para início da coleta considerou-se a zero hora do primeiro dia, analisando-se todos os registros do prontuário de cada paciente referentes ao período de 24 horas. No caso dos pacientes internados no primeiro ou após o primeiro dia de coleta dos dados, foram considerados os registros de cada 24 horas completas na unidade, tomandose como base a hora de admissão do paciente. Dados dos pacientes com 24 horas incompletas, no último dia de coleta, foram desconsiderados no presente estudo.

Foram utilizados os registros efetuados nos impressos: anotação de enfermagem, prescrição de enfermagem, controle de ingeridos e eliminados, impresso de registros específicos (ex: glicemia capilar, saturação de oxigênio), gráficos de sinais vitais e prescrição médica. 
As informações que não se encontravam registradas nos prontuários dos pacientes, como aquelas relacionadas ao tipo e duração de atendimento aos familiares, atividades administrativas e número de pessoas envolvidas, foram obtidas, pelas pesquisadoras, junto aos profissionais que cuidaram dos pacientes, nos momentos de passagem de plantão.

Para a aplicação do NAS aos pacientes classificados como alta dependência de enfermagem foi necessário incluir algumas atividades não contempladas no instrumento original (saturação de oxigênio, glicemia capilar, preparo do corpo, treinamento do cuidador, alimentação oral assistida) mantendo-se, entretanto, a relação lógica com a sua estrutura inicial.

Com a finalidade de uniformizar o significado de cada um dos itens do instrumento e evitar possíveis equívocos de interpretação, foram elaboradas, também, as definições operacionais para sua aplicação aos pacientes deste estudo. Estes procedimentos foram executados pelas pesquisadoras, por meio de consulta às diretrizes para aplicação do NAS, desenvolvidas em estudos realizados em UTI. ${ }^{6,9,11-12}$

Para o registro dos dados coletados foi elaborado um instrumento dividido em duas partes: o primeiro campo destinou-se ao registro das informações relacionadas à identificação do paciente, tais como: iniciais, número de matrícula hospitalar, sexo, idade, data de admissão, diagnóstico médico, procedência e destino. O segundo campo indicava o dia da coleta dos dados, mostrava a representação do NAS e o local específico para o registro da pontuação obtida com a aplicação do instrumento.

Para facilitar a análise dos dados cada paciente recebeu um número de identificação, de forma aleatória, considerando aqueles que já se encontravam na enfermaria no primeiro dia de coleta de dados, que iniciaram com o número um, e depois, sequencialmente, de acordo com a admissão na EAD.

A análise dos dados foi realizada mediante estatística descritiva (freqüência relativa e absoluta) e medida de variabilidade (desvio padrão).

\section{APRESENTAÇÃO E DISCUSSÃO DOS RESULTADOS}

Estiveram internados 22 pacientes na EAD da UCM do HU-USP, durante o período de coleta de dados, sendo dois pacientes classificados como pertencentes às categorias de cuidado intensivo e semi-intensivo e, portanto, retirados da amostra.
Dos 20 pacientes que integraram o estudo, $60 \%$ eram do sexo feminino e $40 \%$ do sexo masculino.

A média de idade encontrada foi de 72,75 anos, com variação de 36 a 95 anos. Os pacientes idosos constituíram a maioria da amostra, com percentual de $85 \%$ com idade igual ou superior a 60 anos e 15\% com idade entre 36 a 59 anos.

Com relação às patologias, verificou-se que $30 \%$ dos pacientes foram internados por alterações neurológicas (Acidente vascular cerebral) e 30\% por alterações respiratórias (descompensação da Doença pulmonar obstrutiva crônica e Broncopenumonia).

Segundo os dados de internação, 70\% das admissões na EAD foram procedentes do Pronto Socorro Adultos (PSA); $25 \%$ de transferência interna, isto é, da própria UCM, por alteração do perfil de complexidade assistencial, de Cuidado Mínimo ou Intermediário para Cuidado de Alta Dependência, e 5\% da Unidade de Terapia Intensiva e Semi-Intensiva Adulto (UTISA).

A média de permanência foi de oito dias com variação de um a 20 dias, sendo que 75\% permaneceram entre um e dez dias. Somente um paciente, que estava internado no início do estudo, permaneceu até o término do período de coleta dos dados.

Em relação ao destino dos pacientes, 35\% obtiveram alta hospitalar; $30 \%$ permaneceram internados até o final da coleta; $20 \%$ foram transferidos internamente por alteração do perfil de cuidado assistencial (cuidado intermediário ou mínimo) e $10 \%$ foram a óbito. Apenas um paciente foi transferido para UTISA (5\%).

\section{Aplicação do NAS}

O NAS foi aplicado a todos os pacientes classificados como alta dependência, internados na EAD da UCM do HU-USP no período da coleta de dados, totalizando 153 medidas, correspondentes a um total de 20 pacientes.

O instrumento demonstrou ser aplicável a esses pacientes, sendo necessário o estabelecimento de diretrizes para a sua aplicação. A clareza do entendimento do instrumento deve ser um consenso entre todos os enfermeiros que o utilizam. Um fator que facilita sua aplicação é o conhecimento da enfermeira sobre o paciente avaliado.

Por ser a coleta dos dados retrospectiva, é imprescindível que o registro das informações de enfermagem seja preciso e retrate as condições do paciente. Dados como o número de profissionais 
envolvidos no cuidado, o tempo despendido para a realização de um procedimento e o suporte dado aos pacientes e familiares, que não são habitualmente registrados na anotação de enfermagem, constituem uma dificuldade para a obtenção dos dados, conforme apontado em outro estudo. ${ }^{9}$

\section{Tempo médio de assistência obtido com a aplicação do NAS}

De acordo com a Tabela 1, observa-se a média do NAS obtido junto a cada paciente integrante do estudo.

Tabela 1 - Pontuação média do NAS dos pacientes de alta dependência de enfermagem, internados na EAD da UCM do HU-USP - 13 de julho a 1 de agosto. São Paulo-SP, 2007

\begin{tabular}{|c|c|c|c|}
\hline $\begin{array}{c}N^{\circ} \text { do } \\
\text { paciente }\end{array}$ & $\begin{array}{l}\text { Média do NAS } \\
\text { por paciente }\end{array}$ & $\begin{array}{l}\text { Média do } \\
\text { NAS }\end{array}$ & $\begin{array}{l}\text { Desvio } \\
\text { Padrão }\end{array}$ \\
\hline 1 & 78,90 & \multirow{20}{*}{51,47} & \multirow{20}{*}{ $\pm 11,17$} \\
\hline 2 & 58,09 & & \\
\hline 3 & 51,52 & & \\
\hline 4 & 63,15 & & \\
\hline 5 & 48,99 & & \\
\hline 6 & 49,96 & & \\
\hline 7 & 45,47 & & \\
\hline 8 & 64,99 & & \\
\hline 9 & 52,23 & & \\
\hline 10 & 34,53 & & \\
\hline 11 & 53,20 & & \\
\hline 12 & 32,57 & & \\
\hline 13 & 51,95 & & \\
\hline 14 & 47,15 & & \\
\hline 15 & 40,20 & & \\
\hline 16 & 40,90 & & \\
\hline 17 & 52,80 & & \\
\hline 18 & 62,71 & & \\
\hline 19 & 40,69 & & \\
\hline 20 & 59,31 & & \\
\hline
\end{tabular}

A pontuação média dos pacientes internados na EAD da UCM do HU-USP foi de 51,47\% (máximo de 78,90 e mínimo de 32,57) e desvio padrão de $\pm 11,17$. Considerando que cada ponto NAS corresponde a 14,4 minutos, ${ }^{9}$ pode-se concluir que o paciente classificado como alta dependência de enfermagem necessita, em média, de 12,3 horas de assistência nas 24 horas (máximo de 18,9 e mínimo de 7,8 horas).

Este tempo supera as horas de assistência de enfermagem preconizados pela Resolução COFEN $\mathrm{N}^{\circ} 293 / 04$ que indica o acréscimo de 0,5 horas de assistência de enfermagem aos pacientes crônicos com idade superior a 60 anos sem acompanhante, classificados como cuidado intermediário $(5,6+$ $0,5=6,1$ horas) e semi-intensiva $(9,4+0,5=9,9$ horas), ${ }^{3}$ embora apresente uma correspondência com esta última indicação quando se subtrai o valor do desvio padrão da média obtida.

O estudo oferece subsídios importantes para o cálculo de pessoal de enfermagem para a categoria de pacientes classificados como alta dependência de enfermagem e corrobora a recomendação de que sejam utilizadas, minimamente, as horas médias preconizadas pelo COFEN para a categoria de cuidado semi-intensivo, na realização da previsão ou avaliação qualitativa e quantitativa de pessoal de enfermagem. ${ }^{2}$

Entretanto, frente à complexidade do tema $\mathrm{e}$ da metodologia utilizada, os resultados apresentados devem ser analisados considerando, também, as limitações do estudo. O tamanho da amostra, o fato de ter sido realizado em apenas uma unidade, de uma única instituição hospitalar, traz restrições para a sua generalização.

Outra limitação refere-se à inexistência de estudos, nacionais e internacionais, desenvolvidos com esta população específica, que impossibilita a análise comparativa dos resultados obtidos.

O estudo evidencia, porém, perspectivas para a realização de novas investigações que explorem o assunto, contribuindo para o estabelecimento de parâmetros referente ao tempo de assistência de enfermagem necessário para o adequado atendimento dos pacientes de alta dependência de enfermagem e, consequentemente, para o aperfeiçoamento do processo de dimensionar pessoal de enfermagem.

A Tabela 2, apresentada a seguir, evidencia as definições operacionais para a aplicação do NAS, bem como a freqüência das pontuações obtidas em cada item e sub-item do instrumento. 
Tabela 2 - Freqüência da pontuação do NAS, obtida pelos pacientes de alta dependência de enfermagem. São Paulo-SP, 2007

(continua)

Definições operacionais para aplicação do NAS em pacientes de alta dependência

f $\%$

1 Monitorização e controles

1a- Sinais vitais, saturação de oxigênio, cálculo e registro do balanço hídrico, pelo menos uma vez por plantão $(4,5)$.

1b- Presença à beira do leito, totalizando duas horas ou mais, para observação ou atividade contínua por razões de segurança ou terapia, como: ventilação mecânica não invasiva, agitação, confusão mental, preparo e administração de fluidos ou medicação, auxílio em procedimentos específicos $(12,1)$

1c- Presença à beira do leito e observação ou atividade contínua por 4 horas ou mais em algum plantão por razões relacionadas aos exemplos acima $(19,6)$.

2 Investigações laboratoriais - coleta de material biológico para investigação laboratorial ou glicemia capilar de horário nas últimas 24 horas $(4,3)$.

3 Medicação - exceto vasoativas; preparo e administração de medicações, independente da quantidade, freqüência e via de administração $(5,6)$.

\section{Procedimentos de higiene}

4 a - procedimentos de higiene tais como: curativos de feridas e cateteres intravasculares, troca de roupa de cama, higiene corporal do paciente em situações especiais; procedimentos especiais, totalizando menos de duas horas contínuas ou somadas, nas 24 horas, necessitando do auxílio de um profissional $(4,1)$.

$4 \mathrm{~b}$ - procedimentos de higiene que durem mais do que duas horas (somadas ou contínuas), nas 24 horas. Curativos complexos, higiene corporal freqüente (três episódios ou mais de diarréia, vômito, incontinência e/ou sangramento), totalizando mais de duas horas ou que exijam o auxílio de dois profissionais, como banho no leito e preparo do corpo em caso de óbito (165).

4c - procedimentos de higiene como curativos complexos, higiene corporal freqüente, acima de quatro episódios de diarréia, vômito, incontinência e/ou sangramento, totalizando quatro horas ou mais de assistência (20).

5 Cuidados com drenos - exceto sonda nasogástrica - $(1,8)$.

6 Mobilização e posicionamento - mudança de decúbito, mobilização do paciente, transferência da cama para a cadeira; mobilização do paciente em equipe.

$6 a$ - realização do procedimento até três vezes nas 24 horas, realizado por um profissional de enfermagem $(5,5)$.

$6 \mathrm{~b}$ - realização desses procedimentos mais do que três vezes em 24 horas ou com dois profissionais em qualquer freqüência $(12,4)$.

$6 c$ - realização desses procedimentos com três ou mais profissionais, em qualquer freqüência, nas últimas 24 horas (17).

7 Suporte e cuidados aos familiares e pacientes - inclui procedimentos tais como telefonemas, entrevistas, aconselhamentos, explicar condições clínicas e tratamentos, lidar com a dor e a angústia, lidar com circunstâncias familiares difíceis.

7a-suporte e cuidado aos familiares e pacientes que requerem dedicação exclusiva por cerca de uma hora em 24 horas. Considerado explicação de procedimentos (4).

$7 \mathrm{~b}$-suporte e cuidado aos familiares e pacientes que requerem dedicação exclusiva por três horas ou mais em 24 horas, contínuas ou fracionadas, em circunstâncias trabalhosas, como treinamento do cuidador para a alta hospitalar (32).

\section{Tarefas administrativas e gerenciais}

8a-tarefas de rotina como: processamento de dados clínicos, solicitação de exames, troca de informações profissionais como a passagem de plantão, visitas clínicas, evolução de enfermagem diária, registros de enfermagem e processamento das transferências ou altas, com duração de até uma hora $(4,2)$. 8b-tarefas administrativas e gerenciais que requerem dedicação integral por cerca de 2 horas nas últimas 24 horas. Incluídos os pacientes que participam de protocolos de pesquisa, que interfiram na rotina da unidade; pacientes ou familiares que recebem orientações de alta hospitalar num período de duas horas não relacionadas ao treinamento do cuidador $(23,2)$.

8c-tarefas administrativas e gerenciais que requerem dedicação integral por cerca de 4 horas nas últimas 24 horas, como: morte com procedimento de doação de órgãos (30). 
Tabela 2 - Freqüência da pontuação do NAS, obtida pelos pacientes de alta dependência de enfermagem. São Paulo-SP, 2007

(conclusão)

\begin{tabular}{lll}
\hline Definições operacionais para aplicação do NAS em pacientes de alta dependência & f
\end{tabular}

9 Suporte respiratório - qualquer forma de ventilação mecânica ou ventilação assistida com ou sem pressão expiratória final positiva, com ou sem relaxantes musculares; respiração espontânea com ou sem pressão expiratória final positiva, com ou sem tubo endotraqueal; oxigênio suplementar por qualquer método $(1,4)$.

10 Cuidados com vias aéreas artificiais - tubo endotraqueal ou cânula de traqueostomia (posicionamento, troca de curativo ou fixação) uma vez ou mais nas 24 horas (1,8).

11 Tratamento para melhora da função pulmonar - espirometria estimulada, terapia inalatória e aspiração endotraqueal $(4,4)$.

12 Medicação vasoativa independente do tipo e dose, nas 24 horas $(1,2)$

13 Reposição intravenosa de grandes perdas de fluídos. Não se aplica pontuação no item $(2,5)$.

14 Monitorização do átrio esquerdo - não se aplica pontuação no item $(1,7)$.

15 Reanimação cardiorrespiratória nas últimas 24 horas (excluído soco precordial) $(7,1)$.

16 Técnicas de hemofiltração - técnicas dialíticas (diálise peritoneal nas últimas 24 horas) $(7,1)$.

17 Medida quantitativa de débito urinário - qualquer medida quantitativa do débito urinário, com ou sem cateteres vesicais, em qualquer duração e freqüência (7).

8 Medida de pressão intracraniana - não se aplica pontuação no item (1,6 ).

19 Tratamento da acidose ou alcalose metabólica complicada nas últimas 24 horas - pacientes que receberam tratamento medicamentoso para a situação $(1,3)$.

20 Hiperalimentação intravenosa - pacientes com nutrição parenteral $(2,8)$.

21 Alimentação enteral - via tubo gástrico ou outra via gastrintestinal) em qualquer freqüência nas 24 horas. Pacientes dependentes de alimentação oral assistida $(1,3)$.

22 Intervenções específicas na unidade - intubação endotraqueal, inserção de marca-passo, cardioversão, endoscopias, cirurgia de emergência no último período de 24 horas, lavagem gástrica, auxílio na passagem de cateter central pela equipe médica (em emergência), sondagem gástrica ou vesical $(2,8)$.

23 Intervenções específicas fora da unidade - encaminhamentos internos ou externos de pacientes para a realização de procedimentos, exames diagnósticos ou cirurgias $(1,8)$.

4529,41

10,65

$-\quad-$

$-\quad-$

$-\quad-$

$-\quad-$

6743,79

$-\quad-$

10,65

- $\quad-$

$57 \quad 37,25$

149,15

$24 \quad 15,69$
A Tabela 2 demonstra que das 153 medidas realizadas, as maiores porcentagens obtidas referiram-se aos itens Medicação, Tarefas administrativas e gerenciais, Procedimentos de higiene, Monitorização e controles, Investigações laboratoriais, Mobilização e posicionamento.

O item Medicação recebeu 100\% da pontuação, pois todos os pacientes receberam algum tipo de medicação durante o tempo em que permaneceram internados.

No item Tarefas administrativas e gerenciais, obtivemos $100 \%$ de pontuação no subitem 8 a. Não obtivemos pontuação nos itens $8 \mathrm{~b}$ e $8 \mathrm{c}$, uma vez que não houve necessidade de dedicação integral por mais de duas horas para a realização de procedimentos administrativos e gerenciais.

Nos itens Procedimentos de higiene e Mobilização e posicionamento, encontramos, respectivamente, $75,82 \%$ das pontuações no subitem $4 \mathrm{~b}$ e $84,97 \%$ no subitem $6 \mathrm{~b}$, pois foram utilizados dois profissionais de enfermagem na sua realização, o que é uma característica comum do paciente classificado como alta dependência de enfermagem. Os subitens 4c e 6c não foram pontuados, pois os pacientes não exigiram mais do que quatro horas de assistência ou do auxílio de três ou mais profissionais para a realização do procedimento.

No que diz respeito ao item Monitorização e controles, $75,16 \%$ da pontuação relacionou-se ao subitem 1a, englobando a verificação de sinais vitais, saturação de oxigênio e registro de líquidos ingeridos e eliminados. No subitem $1 b, 24,84 \%$ da pontuação estava relacionada à necessidade de vigilância devido à agitação, confusão mental e auxílio em procedimentos específicos. O subitem $1 c$ não recebeu pontuação uma vez que caracteriza maior instabilidade, não representando o perfil dos pacientes classificados como alta dependência.

Em relação à Investigações laboratoriais, o estudo mostra que este item recebeu $90,85 \%$ 
das pontuações, evidenciando que a maioria dos pacientes apresentou necessidade de verificação de glicemia capilar ou foi submetido a coleta de exames laboratoriais fora do horário de rotina do serviço de laboratório clínico, ficando, assim, sob responsabilidade da equipe de enfermagem a coleta dos exames realizados.

No Suporte e cuidados aos familiares e pacientes, a pontuação no subitem $7 \mathrm{a}$ e $7 \mathrm{~b}$, justificouse por considerar treinamentos e orientações sobre o cuidado a ser realizado em casa, totalizando $15,69 \%$ das pontuações.

Vale salientar que $30 \%$ das internações foram decorrentes de problemas respiratórios, sendo o item 9, Suporte respiratório, pontuado 33,99\% e o item 11, Tratamento para melhora da função pulmonar, $29.41 \%$.

O item Alimentação enteral recebeu pontuação em 37,25\% dos casos. Foram considerados os pacientes que receberam dieta através de tubo gástrico e a alimentação por via oral assistida, cuja assistência demorava em torno de 15 a 20 minutos. Destes pacientes, cinco necessitaram de auxílio total para alimentação e quatro pacientes fizeram uso exclusivo de alimentação enteral.

Quanto às intervenções específicas realizadas na enfermaria, 9,15\% correspondeu ao auxílio de passagem de cateter central, passagem de sonda vesical de demora e sonda nasogástrica. Com relação às intervenções específicas realizadas fora da unidade, 15,69\% referiram-se à necessidade de encaminhamentos externos e internos, como por exemplo: hemodiálise em outro serviço e encaminhamentos para exames diagnósticos.

No que se refere aos itens Cuidados com vias aéreas artificiais (10), Reanimação cardiorrespiratória (15), Técnicas de hemofiltração (16) e Hiperalimentação intravenosa (20), verifica-se que, embora sejam procedimentos passíveis de ocorrer na assistência aos pacientes de alta dependência de enfermagem, não foram pontuados no período.

Os itens Reposição intravenosa de grandes perdas de fluido (13), Monitorização do átrio esquerdo (14), Medida da pressão intracraniana (18), não se aplicam aos pacientes avaliados no estudo.

Comparando-se os itens e subitens mais pontuados na presente pesquisa, com os resultados apresentados nos estudos realizados em unidades de terapia intensiva, verificamos que, na realidade daquelas unidades, os procedimentos mais pontuados foram os mesmos encontrados na atual investigação., ${ }^{912-13}$ As diferenças encontra- das nas pontuações dos subitens, observadas até mesmo entre estas unidades, sugerem que, além das especificidades de cada grupo de paciente, as características próprias de cada serviço, tais como a estrutura física e organizacional, a política e a filosofia institucional, influenciam a organização do trabalho da equipe de enfermagem e, consequentemente, o tempo de assistência.

\section{CONCLUSÕES}

O NAS demonstrou ser um instrumento aplicável para os pacientes classificados como alta dependência de enfermagem, sendo necessário o estabelecimento de diretrizes para a sua aplicação.

Por ser a coleta de dados realizada de forma retrospectiva, é imprescindível que o registro das informações de enfermagem seja preciso e retrate as condições do paciente. Entretanto, a obtenção de dados referentes ao número de profissionais envolvidos no cuidado, ao tempo despendido para a realização dos procedimentos e ao suporte dado aos pacientes e familiares, que não são habitualmente registrados nos prontuários, constituem uma dificuldade para a aplicação do instrumento.

A pontuação média dos pacientes internados na EAD foi de $51,47 \%$ e o desvio padrão de $\pm 11,17$. Considerando que cada ponto NAS corresponde a 14,4 minutos, ${ }^{9}$ conclui-se que o paciente classificado como alta dependência de enfermagem necessita, em média, de 12,3 horas de assistência, nas 24 horas.

Este tempo supera as horas de assistência de enfermagem preconizadas pela Resolução COFEN $\mathrm{N}^{\mathrm{o}} 293 / 04$, que indica o acréscimo de 0,5 horas de assistência de enfermagem aos pacientes crônicos com idade superior a 60 anos e sem acompanhante, classificados como cuidado intermediário $(5,6$ $+0,5=6,1$ horas) e semi-intensiva $(9,4+0,5=9,9$ horas), ${ }^{3}$ embora apresente correspondência com esta última indicação, quando se subtrai o valor do desvio padrão da média obtida.

Embora o estudo ofereça subsídios para o estabelecimento de parâmetros referente ao tempo de assistência de enfermagem necessário para o adequado atendimento dos pacientes de alta dependência de enfermagem, os resultados apresentados na presente pesquisa devem ser analisados considerando, também, as limitações do estudo. O tamanho da amostra, o fato de ter sido realizado em apenas uma unidade, de uma única instituição hospitalar, traz restrições para a sua generalização. 
A inexistência de estudos, nacionais e internacionais, desenvolvidos com esta população específica, impossibilita a análise comparativa dos resultados obtidos e evidencia a necessidade de novas investigações que explorem o assunto, contribuindo para o estabelecimento de parâmetros adequados para assistir aos pacientes com este perfil assistencial e, consequentemente, para o aperfeiçoamento do processo de dimensionar pessoal de enfermagem.

\section{REFERÊNCIAS}

1. Rogenski KE. Tempo de assistência de enfermagem: identificação e análise em instituição hospitalar de ensino [dissertação]. São Paulo (SP): Universidade de São Paulo. Programa de Pós- Graduação em Enfermagem; 2006.

2. Gaidzinski RR, Fugulin FMT, Castilho V. Dimensionamento de pessoal de enfermagem em instituições de Saúde. In: Kurcgant P, coordenadora. Gerenciamento em Enfermagem. Rio de Janeiro (RJ): Guanabara Koogan; 2005. p.125-37.

3. Conselho Federal Enfermagem. Resolução $N^{\circ}$ 293/2004 [legislação na Internet]. Fixa e estabelece parâmetros para dimensionamento do quadro de profissionais de enfermagem nas unidades assistenciais das instituições de saúde e assemelhados. [acesso em 2007 jun 10]. Disponível em: http:/ / www. corensp.org.br/resolucoes/resolucao293.htm.

4. Fugulin FMT, Silva SH, Shimizu HE, Campos FPF. Implantação do sistema de classificação de pacientes na unidade de clínica médica do Hospital Universitário da Universidade de São Paulo. Rev Med HU-USP. 1994 Jan-Dez; 4(1):63-8.

5. Mello MC, Gaidzinski RR, Fugulin FMT, Marx LC. O desafio gerencial do enfermeiro - adequação do quadro de pessoal [mesa redonda]. Enfermagem Atual. 2003 Maio-Jun; 3(15):6-11.
6. Miranda DR, Nap R, Rijk A, Schaufeli W, Iapichino G, Members of the TISS Working Group. Nursing activities score. Crit Care Med. 2003 Fev; 31(2):374-82.

7. Queijo AF, Padilha KG. Instrumento de medida da carga de trabalho de enfermagem em unidade de terapia intensiva: nursing activities score (NAS). Rev Paul Enferm. 2004 Abr-Jun; 23(2):114-22.

8. Conishi RMY, Gaidzinski RR. Nursing activities score (NAS) como instrumento para medir carga de trabalho de enfermagem em UTI adulto. Rev Esc Enferm USP. 2007 Set; 41(3):346-54.

9. Conishi RMY. Avaliação do NAS - Nursing Activities Score - como instrumento de medida de carga de trabalho de enfermagem em UTI geral adulto [dissertação]. São Paulo (SP): Universidade de São Paulo. Programa de Pós- Graduação em Enfermagem; 2005.

10. Fugulin FMT, Gaidzinski RR, Kurcgant P. Sistema de classificação de pacientes: identificação do perfil assistencial dos pacientes das unidades de internação do HU-USP. Rev Latino-am Enferm. 2005 Jan-Fev; 13(1):72-8.

11. Gonçalves LA, Padilha KG, Souza RMC. Nursing activities score (NAS): proposal for practical application in intensive care unit. Intensive Care Nurs. 2007 Dez; 23(6):355-61.

12. Dias MCCB. Aplicação do Nursing Activities Score - NAS - como instrumento de medida da carga de trabalho em UTI cirúrgica cardiológica [dissertação]. São Paulo (SP): Universidade de São Paulo. Programa de Pós-Graduação em Enfermagem; 2006.

13. Queijo AF. Estudo comparativo da carga de trabalho de trabalho de enfermagem em unidades de terapia intensiva geral e especializadas segundo o nursing activities score (NAS) [tese]. São Paulo (SP): Universidade de São Paulo. Programa de PósGraduação em Enfermagem; 2008. 